\title{
FINITE ELEMENT MODELING OF THE SIGNAL PROPAGATION IN A THIN TUBE AND COMPARISON WITH EXPERIMENTAL DATA
}

\author{
A. Kruisová* , R. Kolman*, J. Trnka*, M. Mračko*
}

\begin{abstract}
In finite element modeling of wave propagation problems, both the spatial and temporal discretization lead to dispersion errors. It means that the phase velocity of propagated wave is related to its frequency. In framework of temporal-spatial dispersion analysis, the time step size for implicit time integration method based on the Newmark method is proposed for linear and quadratic serendipity plane finite elements. In this paper, we verify the theoretical dispersion analysis by elastic wave propagation in thin tube, where experimental results are known. Such time step size was used in finite element modeling of the stress wave propagating in this thin steel tube, the results of simulations were compared with experimental results.
\end{abstract}

\section{Keywords: wave propagation, finite element method, dispersion errors}

\section{Introduction}

Setting of the size of an element and the size of the time step are crucial for the modeling of the wave propagation by finite elements method (FEM). It is known, that the FE mesh behaves as a frequency filter, the higher frequencies cannot be transmitted through the numerical model and all numerical simulations are influenced by the dispersion error, see Mullen and Belytschko (1982) and Schreyer (1983). The suitable mesh size of the linear and quadratic serendipity plain strain finite element was established in Kolman et al (2013). Expecting the spatial dispersion errors approximately $2 \%$, the size of linear and quadratic elements with respect to the minimum wave length of the propagated wave $\lambda$ is $H / \lambda=1 / 10$ and $H / \lambda=1 / 3$, resp., $H$ is the length of the finite element edge. Kolman et al (2016) gives the temporal dispersion errors for explicit time integration method based on the central difference method. In a similar way, the dispersion errors was derived for the implicit Newmark method. Here, the established time step size is verified on an example of wave propagation through the thin tube. The numerical results are compared with experimental data.

\section{Dispersion errors}

We extended the full (both spatial and temporal) dispersion analysis of the finite element method for implicit time integration based on the Newmark family, see Kruisova et al (2018). The numerical strategy for evaluation of dispersion relationships has been adopted from work of Kolman et al (2016). In this paper, we present several polar dispersion graphs for linear and quadratic serendipity plane finite element method for different dimensionless time step size given by the Courant number defined as $C=\Delta t c_{1} / H$, where $\Delta t$ is the time step size and $c_{1}$ is the longitudinal elastic wave speed.

In Fig. 1, the temporal-spatial errors in sense of polar diagrams for the linear and quadratic elements are illustrated for the selected Courant numbers of interest $C=\{\rightarrow 0,0.1,0.5,1.0\}$ and Poisson ratio $\nu=0.3$. The dashed line circles shows the theoretical values for the phase velocity of the longitudinal wave (small circle) and shear wave (bigger circle) in plain strain continuum. The solid lines are the ratio of the numerical

\footnotetext{
Ing. Alena Kruisová, Ph.D.: Institute of Thermomechanics, CAS, Dolejškova 1402/5; 182 00, Prague; CZ, alena@it.cas.cz Ing. Radek Kolman, Ph.D.: Institute of Thermomechanics, CAS, Dolejškova 1402/5; 182 00, Prague; CZ, kolman@it.cas.cz Ing. Jan Trnka, CSc.: Institute of Thermomechanics, CAS, Dolejškova 1402/5; 182 00, Prague; CZ, trnka@it.cas.cz Ing. Michal Mračko, CSc.: Institute of Thermomechanics, CAS, Dolejškova 1402/5; 182 00, Prague; CZ, mracko@it.cas.cz
} 
phase velocity $c^{h} / c_{1}$ for the longitudinal (bigger circle) and shear waves (smaller circle), resp. The other solid lines in the right plot for the quadratic elements show the spurious (optical) branches of dispersion spectrum. The dispersion error (the sum for the spatial and temporal dispersion) is for the Courant number $C=0.1$ approximately $3 \%$ for the linear elements and $4 \%$ for the quadratic elements. The worst values of the dispersion error occur when the wave propagates in the diagonal direction of the mesh, smaller dispersion errors occur when the wave propagates in the direction of the edge of the element

$H / \lambda^{h}=1 / 10$, linear elements

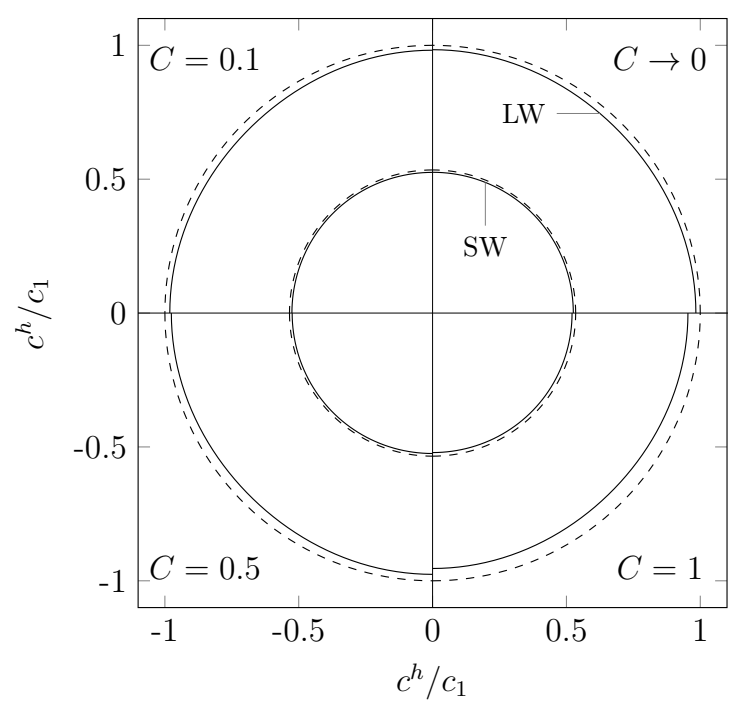

$H / \lambda^{h}=1 / 3$, quadratic elements

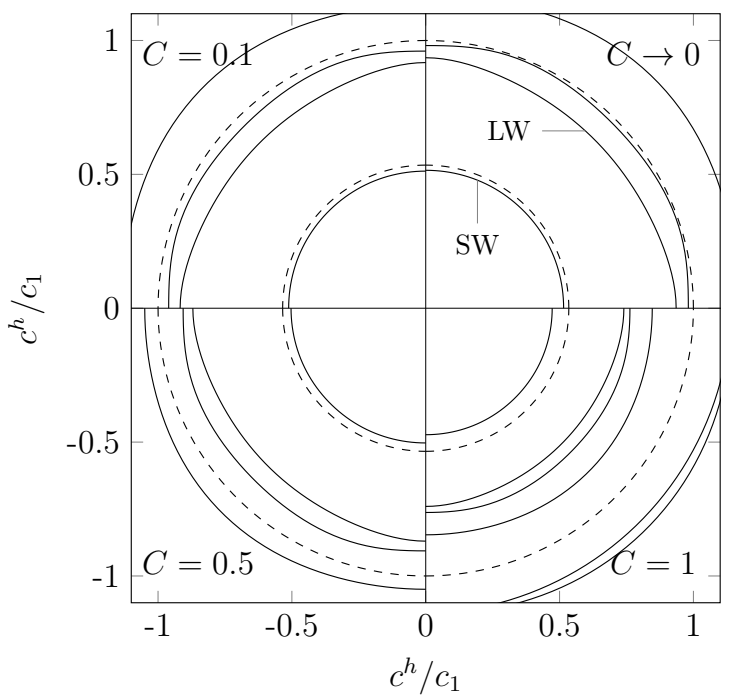

Fig. 1: The normalized dispersion errors for linear and quadratic elements for different values of Courant number $C$. The directional relation of the phase velocity dispersion error for the shear wave is plotted with solid line (small circle) and is denoted by SW, the theoretical value in continuum corresponds to the smaller dashed circle. For the longitudinal wave, the directional relation of the phase velocity dispersion error is plotted with solid line, bigger for the bi-linear elements and the second smallest for the quadratic elements and is denoted by LW. The bigger dashed circle denotes the phase velocity for the longitudinal wave. The other solid lines for the quadratic element denote the optical modes.

\section{Experimental results}

To illustrate the choice of the time step step $\Delta t$ for the Newmark integration scheme, the stress signal propagating through a thin steel tube was measured and simulated by the finite element method. The length of the tube was $610 \mathrm{~mm}$ and the internal and external diameters were $101.5 \mathrm{~mm}$ and $108 \mathrm{~mm}$, resp. The stress signal propagating through the tube was induced by the flat spring with the diameter of the button 8 $\mathrm{mm}$. The mechanical properties of the steel used in calculation were the Young modulus $E=210 \mathrm{GPa}$, the Poisson ratio 0.3 , and the density $\rho=7800 \mathrm{~kg} \cdot \mathrm{m}^{-3}$. The tube was loaded in the axial direction at the point A at the cross section, see the scheme at Fig. 2, and the input signal (contact force) was measured close to this point. The output signal (normal component of velocity) was measured in the axial direction at the cross section in the other end of the tube in two points, B and C by Polytec vibrometer CLV-2000.

Measured signals at the points $A, B$ and $C$ are also given in Fig. 2. The maximum loading force is $740 \mathrm{~N}$ and the time duration of loading is approximately $30 \mu \mathrm{s}$. Since the measured loading pulse is characterized by small oscillations, it was smoothed for the computation purposes, only 25 highest frequencies of the spectra was used in the inverse Fourier transformation.

\section{FEM calculations}

Since the periodic finite element mesh serves as a frequency filter for higher frequencies, the size of the mesh has to be adjusted to the highest frequency in loading pulse, which was in the smoothed signal equal to $f_{\max }=60 \mathrm{kHz}$. The suitable mesh size $H$ of an element was proposed in Kolman et al (2013), for the square quadratic element is $H<0.33 c_{2} / f_{\max }, c_{2}$ is the velocity shear waves in continua, this size of an 


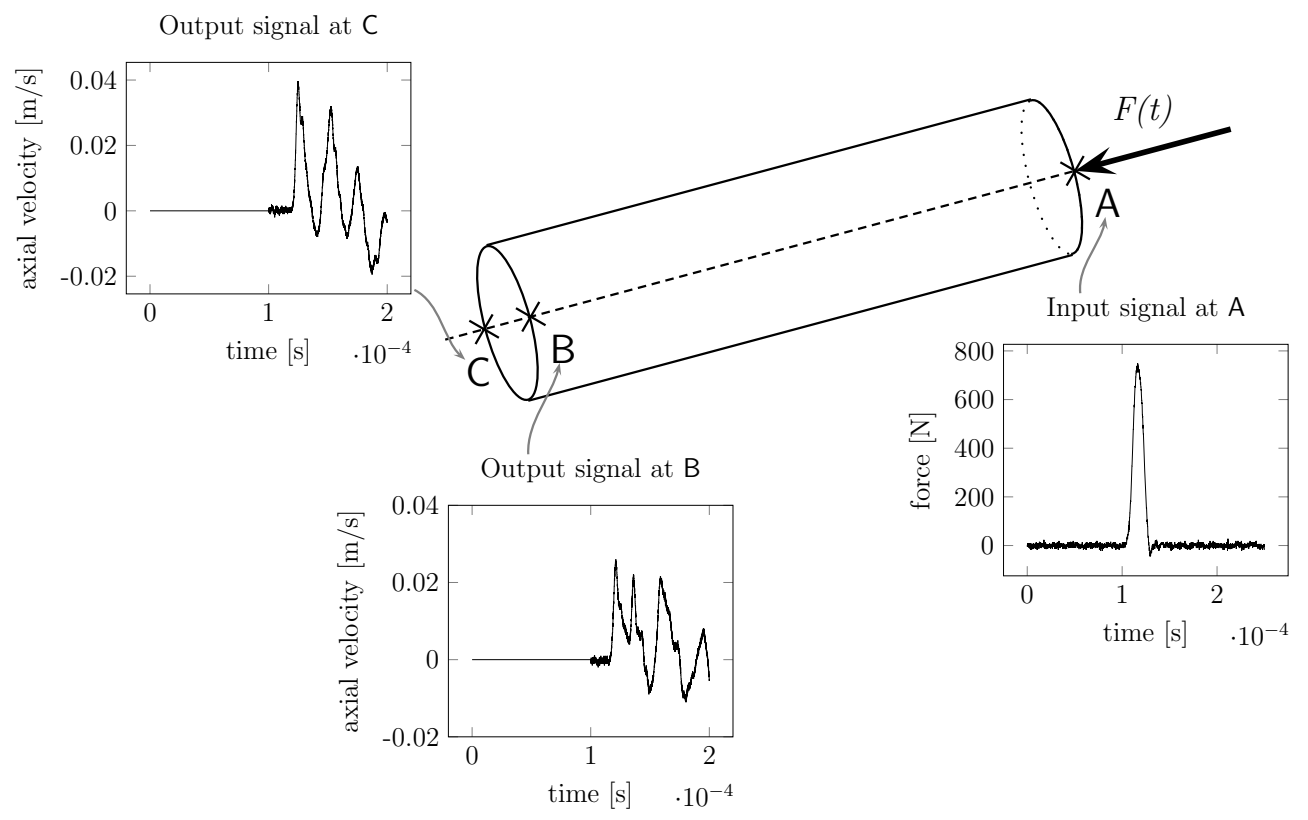

Fig. 2: Experimental setup

element corresponds to the model when a wavelength is modeled by 3 elements, and six nodes. For linear finite element mesh, ten elements are needed for modeling of one wavelength, so the size of the element is given by $H<0.1 c_{2} / f_{\max }$. Using material properties of the steel tube given above the minimum size of an quadratic element is $17 \mathrm{~mm}$, for an linear element $5 \mathrm{~mm}$. With respect to the thickness of the tube, we used an quadratic element $5.5 \mathrm{~mm} \times 5.5 \mathrm{~mm}$ (one element to the thickness) and a linear element of $4 \mathrm{~mm} \times 4 \mathrm{~mm}$ (two elements to the thickness).

For the time integration the average acceleration method from the Newmark family, see Newmark (1959), was used with parameters $\gamma=0.5$ and $\beta=0.25$, see Hughes (2000). At the point $A$ the time history of the force is prescribed, the normal components of velocity of both points $B$ and $C$ are stored. The finite element code PMD was used, PMD (2002).

In Fig. 3 the results of the FE simulations together with measured signals are given. The upper plots are for the linear mesh elements of the size $H=4 \mathrm{~mm}$, which corresponds to $H / \lambda=0.07$, the bottom plots are for the quadratic mesh with the element of the size $H=5.5 \mathrm{~mm}$, which corresponds to $H / \lambda=0.1$. The numerical simulations have been performed for the time step size given by different Courant numbers. Based on comparison of numerical results with experimental data, one can see very good agreements for smaller time step size up to $C=1.0$ for linear FEM and to $C=0.5$ for quadratic FEM. For larger Courant number, the results are polluted by dispersion errors. Here, the numerical wave speeds in FE model are underestimated.

\section{Conclusions}

In this work, we have verified the full dispersion analysis of linear and quadratic serendipity FEM method with implicit time integration by a real wave propagation experiment. Also relationships for suitable setting of mesh size and time step size for knowledge of maximum loading frequency have been tested. Based on these realized simulations in this work, one can use these nominated suggestions in real FE simulations of elastic wave propagation problems. In future, the time reversal modeling will lead to the detailed analysis of the dispersion errors in numerical FE simulations.

\section{Acknowledgments}

The work of A. Kruisová and R. Kolman was supported by the grant project 17-22615S of the Czech Grant Agency within the institutional support RVO: 61388998. The work J. Trnka and M. Mračko was supported by the Centre of Excellence for Nonlinear Dynamic Behaviour of Advanced Materials in Engi- 

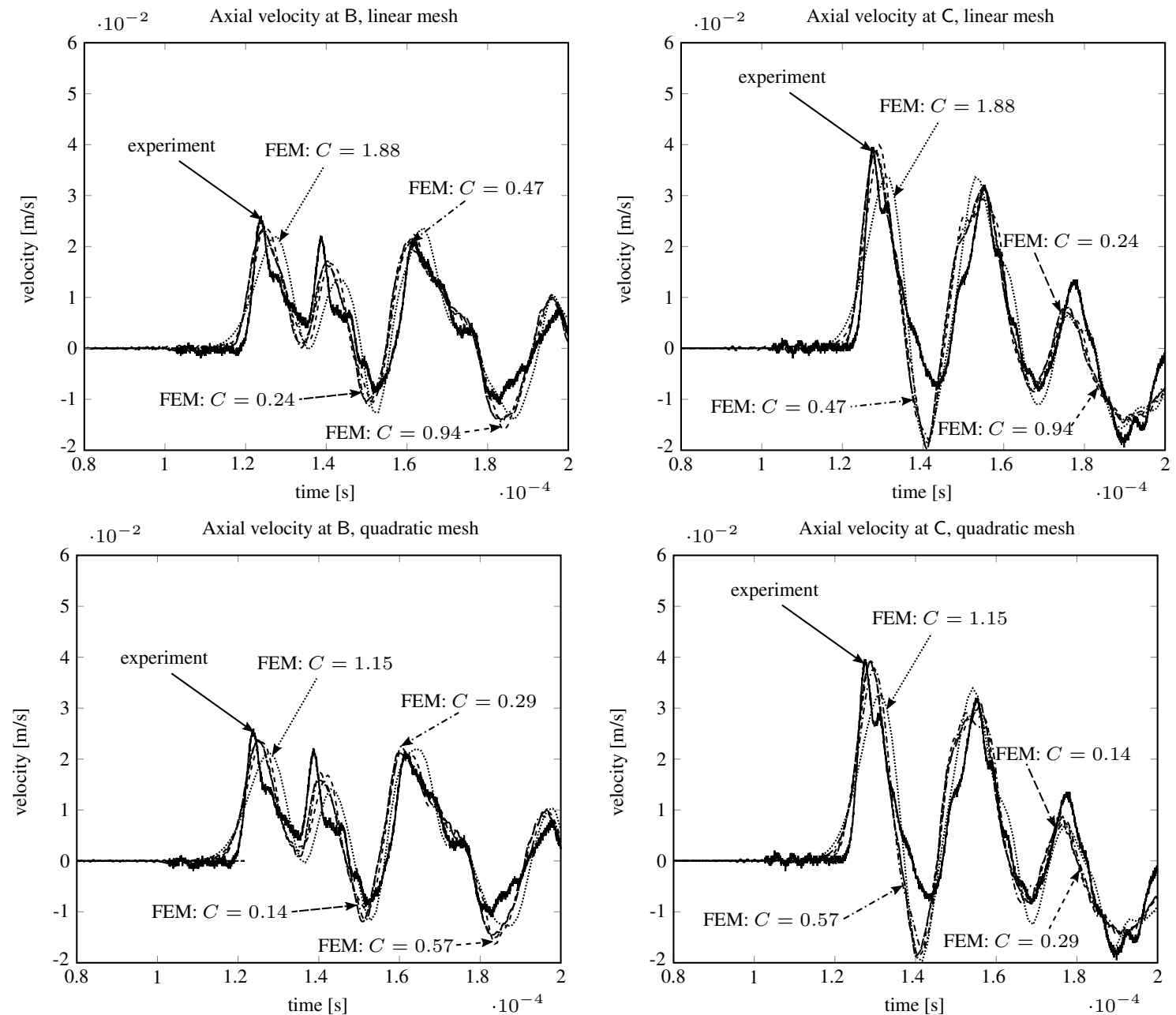

Fig. 3: Comparison of the experimental results with computational simulations for different time steps given by different Courant numbers. The upper plots are for the linear mesh, the bottom plots are for the quadratic mesh. Left side graphs give the results in point $B$, which is at the same side of the tube, as the loading was placed, the right side graphs show the result at the point $C$, which is on the opposite side of the tube, than the loading place.

neering CZ.02.1.01/0.0/0.0/15_003/0000493 (Excellent Research Teams) in the framework of Operational Programme Research, Development and Education.

\section{References}

Hughes, T.J.R., (2000), The Finite Element Method: Linear Static and Dynamic Finite Element Analysis, Dover Publications, New York.

Kolman, R., et al., (2013), Grid dispersion analysis of plane square biquadratic serendipity finite elements in transient elastodynamics. International Journal for Numerical Methods in Engineering, Vol 96, pp 1-28.

Kolman R., et al., (2016), Temporal-spatial dispersion and stability analysis of finite element method in explicit elastodynamics. In: International Journal for Numerical Methods in Engineering, Vol 206, pp 113-128.

Kruisova, A., et al., (2018) Temporal-spatial dispersion of finite element method in implicit elastodynamics. International Journal for Numerical Methods in Engineering, under preparation.

Mullen, R., Belytschko, T., (1982), Dispersion analysis of finite element semidiscretizations of the two-dimensional wave equation. International Journal for Numerical Methods in Engineering, Vol 18, pp 11-29.

Newmark, N., (1959) A method of computation for structural dynamics. Journal of the Engineering Mechanics Division, Vol 85, No 3, pp 67-94.

PMD, FEM program, (2002) Vamet s.r.o., version.f77.7.

Schreyer, H.L. (1983), Dispersion of semidiscretized and fully discretized systems. In: Computational Methods for Transient Analysis, (Belytschko T. and Hughes T.J.R., eds). North-Holland: Amsterdam; pp. 267-299. 